\title{
Gradhiva
}

GRADHIV

Revue d'anthropologie et d'histoire des arts

16 | 2012

Chines, l'État au musée

\section{Le nationalisme dans les musées locaux à Taïwan : colonisation, autoritarisme et démocratie}

Nationalism in Taiwanese Local Museums: Colonisation, Authoritarianism and Democracy

Lee Wei-I

\section{OpenEdition}

\section{Journals}

Édition électronique

URL : http://journals.openedition.org/gradhiva/2461

DOI : 10.4000/gradhiva.2461

ISSN : 1760-849X

Éditeur

Musée du quai Branly Jacques Chirac

Édition imprimée

Date de publication : 1 décembre 2012

Pagination : 64-83

ISBN : 978-2-35744-048-7

ISSN : 0764-8928

\section{Référence électronique}

Lee Wei-I, « Le nationalisme dans les musées locaux à Taïwan : colonisation, autoritarisme et démocratie », Gradhiva [En ligne], 16 | 2012, mis en ligne le 01 décembre 2015, consulté le 30 avril 2019. URL : http://journals.openedition.org/gradhiva/2461 ; DOI : 10.4000/gradhiva.2461 


\section{Le nationalisme dans les musées \\ locaux à Taïwan : colonisation, autoritarisme et démocratie}

par Lee Wei-I

Le présent article se propose d'analyser, à travers l'examen de l'histoire du développement des musées locaux à Taïwan, le lien entre la construction culturelle et l'identité collective, né de la rencontre entre la société locale et le nationalisme. L'analyse se déploie sur deux axes principaux: d'abord, dans la perspective d'une anthropologie politique sur les États-nations, l'auteur s'efforce de repérer, à travers les trois vagues de construction des musées locaux resituées dans leur contexte social, les différentes formes d'intervention de l'État dans le local, et par là tente d'expliciter la façon dont le politique s'imbrique dans la représentation culturelle sous différents régimes politiques; ensuite, dans la mesure où Taïwan reste aujourd'hui encore prise dans un conflit inextricable entre de multiples formes de nationalisme, l'auteur essaie, dans le cadre d'une réflexion sur le lien entre l'imaginaire culturel et la formation de l'État, d'explorer le phénomène qu’il appelle «l'absence de la société » dans la construction des musées locaux, enlisée dans un complexe identitaire au sein même du jeu démocratique. 


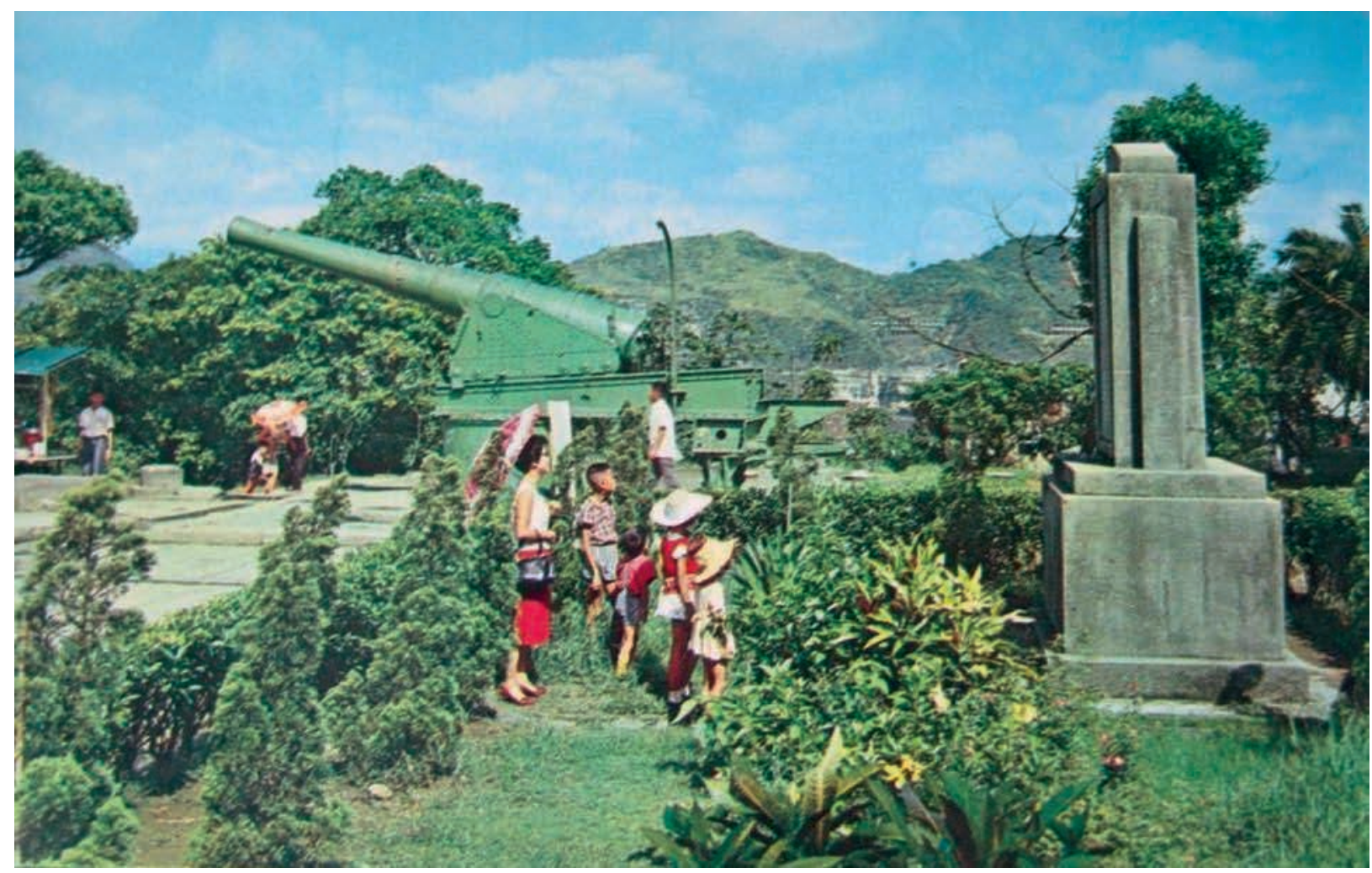

fig. 1

Famille japonaise (?) devant une stèle dédiée à la mémoire du Prince Kitashirakawa à Keelung (Jilong), années 1940. Collection Marc Plumb.

1. 總督府博物, Sotokufu Hakubutsukan: premier grand musée polyvalent moderne fondé en 1908 par le gouvernement colonial japonais, il fut le plus important musée de l'île jusqu'à la fin de la guerre, où il fut rebaptisé musée provincial de Taïwan.

\section{Introduction}

Quand les instances du pouvoir étatique mettent en scène l'imaginaire politique à travers les objets, on assiste à un processus d'institutionnalisation de la représentation collective, dont une des formes modernes majeures est le musée. Des études ont montré que, dans la mutation des sociétés traditionnelles en États-nations modernes, l'idéologie nationaliste se manifeste pleinement à travers la construction des musées; sachant que dans les pays émergents de l'après-guerre, et notamment dans les anciennes colonies des empires, le développement des musées est étroitement lié à la décolonisation, à la construction de l'État, ainsi qu'à la fabrication d'une identité nationale (Kaplan 1994; Poulot 1997; Thiesse 1999; Anderson 1991; Collomb 1999; Macdonald 2003). Taïwan ne déroge pas à la règle. Le développement centenaire des musées y répond à un remodelage politique permanent en fonction des configurations historiques et politiques successives - colonisation japonaise, régime dictatorial et autoritariste, démocratisation. Plusieurs études se sont penchées sur les liens entre le colonialisme japonais et le musée du Gouverneur ${ }^{1}$ ainsi que sur les multiples foires et expositions de l'époque coloniale (Lu 2005; Li 1997); nousmême avons examiné dans une précédente étude les interactions entre le nationalisme et l'institutionnalisation des musées à Taïwan pendant la période de la décolonisation et de la guerre froide (Lee 2007b), et esquissé ailleurs un aperçu de l'évolution des imaginaires collectifs dictés par les conditions politiques dans la création des grands musées depuis cent ans (Lee 2007a).

Ces études ont procédé essentiellement par l'analyse des actions «nationales» de l'appareil politique, mettant en lumière les liens historiques 
entre le colonialisme, le nationalisme et les grandes institutions muséales. Mais la construction de l'imaginaire collectif ne se cantonne pas dans la haute sphère nationale; le pouvoir, toujours désireux de mettre sous contrôle toute autonomie, cherche souvent à mener cette construction jusqu'au niveau local. Or cette tension entre national et local se décline de façon d'autant plus complexe dans le cas de Taïwan, qui jouit d'un statut problématique de nation-localité. La construction des musées «locaux» est en cela révélatrice des frontières mouvantes des imaginaires collectifs sur l'île. II est évident que l'impérialisme colonial japonais, le nationalisme impérialiste chinois et enfin le (néo)nationalisme démocratique ont fortement marqué ces entreprises muséales: les trois vagues de construction, correspondant chacune à un régime politique, constituent ainsi la trame principale de cette réflexion.

La première vague apparut dans les années 1930, lors des campagnes “d'éducation du terroir» (鄉土教育運動, xiangtu jiaoyu yundong) [Chou 1997] et de «préservation des sites historiques et des monuments naturels de Taïwan» (臺灣史蹟名勝天然紀念物保存運動, Taïwan shiji mingsheng tianran jiniangwu baocun yundong) [Lee 2006], avec la création d'un ensemble de musées dit «du terroir» dans plusieurs localités, dont Jilong, Taidong, Hualian et Tainan. La deuxième vague intervint surtout dans les années 1970, alors que l'impact provoqué dans la société par l'essor économique semblait inspirer des mouvements de protection des monuments historiques et l'inauguration d'un certain nombre de musées d'histoire ou de commémoration «locale». La troisième vague débute avec le processus de démocratisation des années 1990, entraînant la création de nombreux musées locaux aux dimensions variées, ainsi que de musées ethniques et communautaires.

Nous proposons ici, en suivant les étapes de ces édifications, d'analyser le lien entre la construction d'un espace culturel et l'identité collective, né de la rencontre entre la société locale et le nationalisme. Nous nous concentrerons sur deux axes principaux.

D'abord, dans la perspective d'une anthropologie politique des États-nations, nous nous efforcerons de repérer, dans le cas de l'île de Taïwan - en resituant les trois vagues de construction des musées locaux dans leur contexte social au sein de cette entité politique sous l'influence de multiples impérialismes depuis quatre cents ans -, les différentes formes d'intervention de l'État dans le local sous les trois régimes politiques de la colonisation, de la dictature et de la démocratie. Par là, nous essaierons d'expliciter le rôle de certains éléments historiques dans la façon dont le politique s'imbrique dans la représentation culturelle.

Ensuite, dans la mesure où Taïwan reste aujourd'hui encore prise dans un conflit inextricable entre de multiples formes de nationalisme, nous tenterons, dans le cadre d'une réflexion sur le lien entre l'imaginaire culturel et la formation de l'État, de cerner le phénomène désigné comme «l'absence de la société » dans la construction des musées locaux, enlisée dans un complexe identitaire au sein même du jeu démocratique. 
2. Inspirée des modèles américain et allemand,

l'éducation nationale moderne au Japon a connu à partir des années 1910 une phase de "localisation " des programmes scolaires par l'édition de nombreux manuels d'éducation du terroir, et ce mouvement généralisé dans la métropole a gagné la colonie vers la fin des années 1920.

3. Nous soulignons.

\section{ci-contre}

fig. 2

Statue de Koxinga (Zheng Chenggong) érigée en 1961, œuvre du sculpteur Yuyu Yang (1926-1997) @ Central News Agency, CNA.

\section{Colonialisme impérial du Japon et localité taïwanaise:} l'amour du terroir sans l'histoire locale (les années 1930)

À l'époque coloniale japonaise, les différentes manifestations politico-culturelles comme les foires et les expositions, nationales ou universelles, faisaient de Taïwan dans son ensemble une localité à «montrer " auprès du Japon métropole (Lu 2005). Toutefois, des initiatives proprement locales de construction muséale firent rapidement leur apparition sur l'île, essentiellement sous forme de lieux d'exposition des produits locaux. C'est le cas de la salle d'exposition des Produits du district de Tainan, inaugurée en 1899, qui se transforma en musée de Tainan à partir de 1902.

Conformément à l'idée initiale de la colonie comme réserve en ressources naturelles, les localités étaient avant tout présentées comme des régions pourvoyeuses de richesses variées. Ce trait fondamental était encore perceptible dans la restructuration du musée de Tainan en 1923, divisé en deux institutions, l'une à caractère fortement naturaliste, l'autre tournée vers l'économie: le musée municipal de l'Éducation de Tainan, avec ses spécimens d'oiseaux tropicaux, et la salle d'exposition municipale des Marchandises de Tainan. Ce n'est qu'à partir des années 1930 que l'imaginaire local, mis en avant dans les musées, se déplaça des ressources agricoles et aquatiques, de l'économie marchande et des sciences naturelles vers des représentations culturelles. Le contrôle stabilisé de la colonie n'étant pas acquis, les autorités politiques comptaient sur une franche redéfinition politique de l'espace «local» qu'était Taïwan. Une série de manœuvres officielles furent ainsi tentées, notamment la Campagne d'éducation du terroir ${ }^{2}$. En matière de manifestation de la mise en représentation culturelle, on peut noter l'organisation de la grande Exposition commémorative pour le tricentenaire de la culture taïwanaise en 1930 ou l'ouverture du musée d'Archives de Tainan en 1932, rebaptisé musée municipal d'Histoire de Tainan en 1937, ou encore la publication de listes des monuments historiques classés dans l'île.

Pour cerner la teneur exacte de ces entreprises à visée culturelle, il suffit de nous pencher sur certains détails tout à fait instructifs. Prenons le cas de l'Exposition commémorative pour le tricentenaire de la culture taïwanaise organisée en 1930. Le texte portant sur les "Archives tricentenaires de Taïwan» rédigé pour l'occasion par Yamanaka Shou (山中樵, 1882-1947), à l'époque directeur de la bibliothèque de la maison du Gouverneur, retient par exemple notre attention. L'intention des Japonais y est en effet dévoilée sans détour à travers l'évocation du sort qu'il conviendrait de réserver à l'une des figures majeures de l'histoire de Taïwan, le général chinois Cheng Ch'eng-kung (鄭成功, “Koxinga», 1624-1662): “Les faits et gestes des trois générations des Cheng, constate l'auteur, laissent des traces profondes dans l'esprit des Taïwanais. Pour les éliminer de la mémoire des Taïwanais $^{3}$, nous devons absolument reconsidérer notre attitude vis-à-vis de l'histoire et de ses vestiges..." (Ts'ai 1984) II était donc impératif de dépasser la conquête des Cheng comme point de départ consacré de ladite culture «taïwanaise». L'attention était alors redirigée sur la domination hollandaise, éphémère mais antérieure. Ainsi le «tricentenaire" se référait-il à la date de 1631, l'année où les Hollandais ont achevé la construction du fort Zeelandia à Tainan, événement présenté comme fondateur dans l'histoire insulaire. 


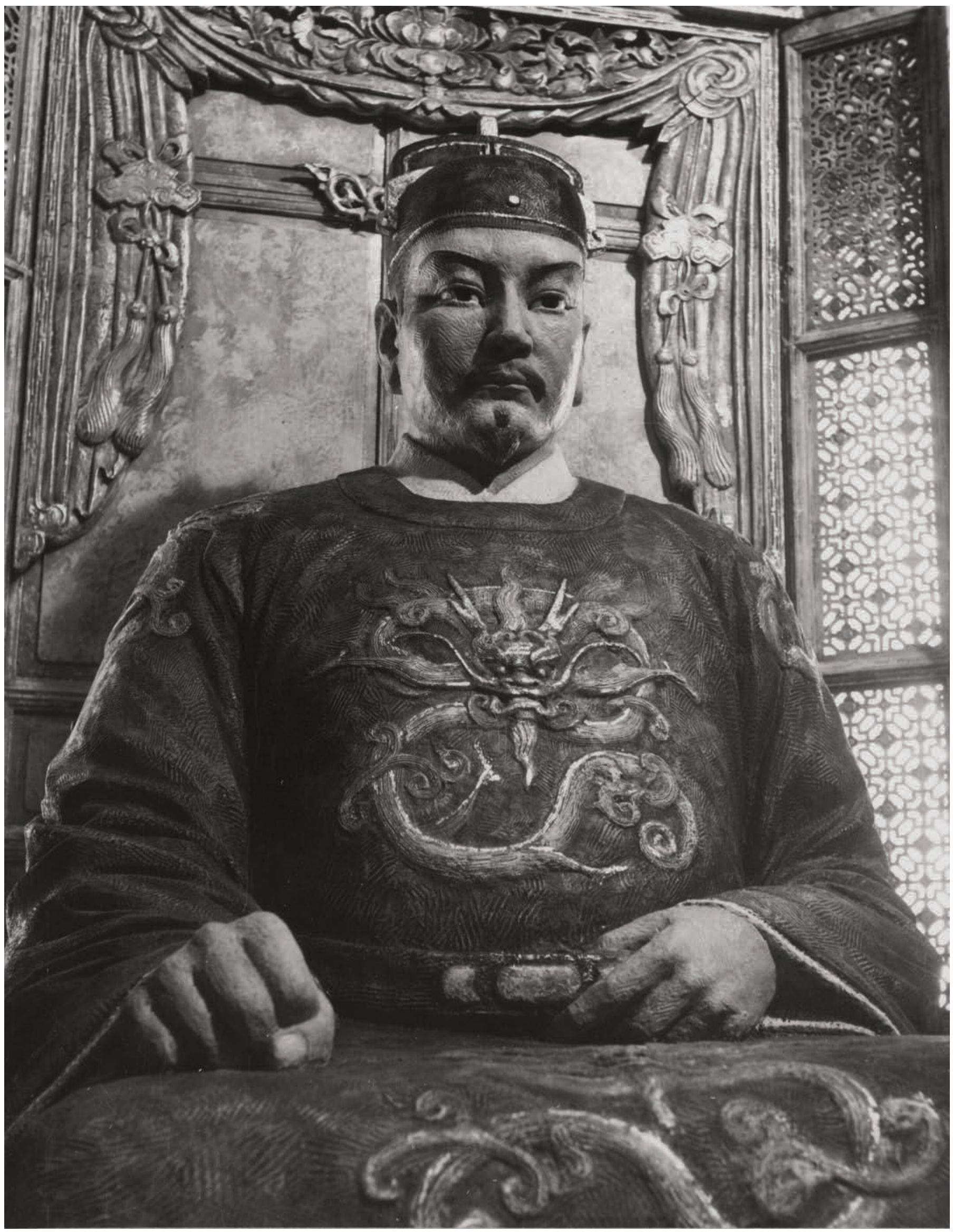


Une campagne de sensibilisation de l'opinion avait accompagné l'inauguration de l'exposition, annoncée par de nombreux articles de presse. Le Nouveau Journal de Taïwan, premier organe de presse à l'époque, avait même publié dans ses colonnes, sous forme d'une série de reportages, la traduction de l'ouvrage Formose négligée ('T Verwaerloosde Formosa en hollandais), écrit en 1675 par le dernier gouverneur hollandais Frederik Coyett (1615-1687). Par ce rappel presque nostalgique de la présence hollandaise sur l'île, les Japonais affirmaient leur ambition de remédier à cette déplorable «négligence» dans l'histoire de Taïwan et cherchaient à instaurer une nouvelle perspective japonaise sur cette histoire.

L'établissement des listes de monuments et sites classés allait dans le même sens. Entre 1933 et 1941, la maison du Gouverneur de Taïwan rendit publiques trois Listes des vestiges historiques, sites célèbres et monuments naturels de Taïwan, classés en plusieurs catégories: nature, animaux, botanique, géologie, vestiges historiques et sites célèbres. Inspirée des pratiques américaines et européennes contemporaines, la démarche affichait bien l'intention de préserver et de protéger les sites «historiques» de Taïwan, mais on percevait très vite qu'il s'agissait surtout de mettre en valeur une certaine version de l'histoire insulaire. Sur les soixante-treize lieux sélectionnés dans la catégorie des vestiges historiques et sites célèbres, trente-huit étaient liés au passage sur l'île du prince Kitashirakawa Yoshihisa 4 - ses champs de bataille, ses camps militaires, ses lieux de repos... -, et treize à la campagne militaire japonaise et au passage des généraux japonais. Seuls vingt-deux sites rassemblaient des vestiges archéologiques, des forts, d'anciennes portes de ville et des monuments culturels représentatifs d'autres périodes historiques, allant de la préhistoire à l'empire des Qing en passant par la période des Hollandais et le royaume des Cheng.

Cet inventaire nous montre que l'ordre temporel et les lieux de mémoire politique de la conquête japonaise de Taïwan étaient le véritable enjeu de la construction d'une conscience «historique» orchestrée par le gouvernement colonial. Grâce au classement de la cinquantaine de vestiges de guerre et monuments témoins de l'histoire de la conquête militaire de l'île par le Japon, c'est toute l'île qui fut requadrillée à travers le prisme temporel japonais, et par là même inscrite dans une chronologie préétablie de l'histoire moderne japonaise. Et si les monuments historiques incontournables des autres périodes ont été eux aussi classés, c'était moins en tant que témoins de l'histoire taïwanaise qu'à titre de signes du passé mis en contraste avec I'histoire moderne japonaise qu'il s'agissait de valoriser.

La création de musées locaux à Taïwan durant les années 1930 se fit en fonction de la même configuration historique. L'ouverture du musée du terroir de Jilong en 1934, par exemple, faisait suite à une commémoration coloniale de l'année précédente. Après l'inauguration en 1933 d'un mémorial du passage du prince Yoshihisa à Jilong, le gouvernement local jugea bon de faire classer ce site, marqué par un court séjour (une semaine) du prince, et décida de restaurer le campement de la garnison qui devint, un an plus tard, le musée. Quant au musée du terroir de Taidong et à celui du port de Hualian, ils s'étaient installés dans les locaux de l'Exposition commémorative du quarantième anniversaire de la nouvelle ère politique organisée en 1935. 
Comme l'a montré l'historienne Chou Wan-Yao dans son étude sur la Campagne d'éducation du terroir, la logique, évidente au Japon métropole, qui veut que «l'amour du terroir mène à l'amour de la patrie» ne pouvait fonctionner dans la colonie qu'était Taïwan sans une contorsion imposée à l'idée même du terroir. À partir d'une analyse des manuels de langue japonaise utilisés dans les écoles primaires, Chou démontre que, pour transformer l'amour spontané des colonisés pour leur pays en un patriotisme nationaliste, les manuels travaillaient en effet à éliminer la conscience identitaire historique des colonisés pour lui substituer l'histoire de la métropole colonisatrice, et par là assimiler le sentiment d'appartenance locale des Taïwanais à une identité nationale japonaise (Chou 1997). Les musées locaux à Taïwan, dits "du terroir ", construits dans le même esprit visaient en effet à intégrer des espaces et des patrimoines taïwanais dans l'ordre temporel de la domination japonaise afin de les réinvestir dans la construction d'un imaginaire national.

\section{Nationalisme chinois autoritariste et localités taïwanaises le recours à l'Empire (les années 1970)}

Jusqu'au milieu des années 1960, dans une Taïwan placée sous un régime dictatorial, confrontée à une situation politique et économique encore instable, la construction des musées sur l'île se concentra sur les grandes institutions nationales: décolonisation du musée du Gouverneur, création du musée national d'Histoire ou réouverture du musée du Palais. Sur le plan local, reprenant certaines collections des anciens musées japonais du terroir placées sous le contrôle de la bureaucratie éducative de la province, des maisons provinciales de l'éducation sociale, simples relais administratifs destinés à accueillir les expositions et les manifestations politiques, faisaient office de musées locaux (Lee 2008: 207-234).

Mais dans les deux décennies suivantes, et surtout dans les années 1970, on vit apparaître une vingtaine de petits musées, certains thématiques, consacrés à la fonction publique comme le musée de la Poste ou le musée de la Réforme agraire, d'autres dédiés à l'enseignement et à la recherche comme le musée Huagang de l'université de la Culture chinoise, et d'autres encore centrés sur la culture locale comme le musée du Patrimoine folklorique de Lugang et le musée du Patrimoine historique du district de Tainan. Cet essor des petits musées était certainement lié au développement de l'île. Les décennies 1960 et 1970 furent en effet celles d'un changement rapide et radical qui modifia la structure économique et sociale du pays. Les bouleversements ne manquèrent pas d'affecter les équilibres traditionnels et de susciter le désir, parfois angoissé, de les préserver ou de les restaurer. Les importants mouvements de la littérature et de l'art, dits à nouveau «du terroir ", prônant le retour à la terre et à la tradition qui se développèrent dans les années 1970 furent précisément une réaction à l'anxiété générée par la modernisation.

Ce qui faisait la particularité de Taïwan était que ce conservatisme culturel en réaction à l'industrialisation coïncidait avec l'entrée de l'île dans l'isolement politique international après l'éviction de la république de Chine des Nations unies. Favorisée par la réussite économique, et en attendant l'ouverture politique des années 1980, la tentation du repli s'affirmait
4. Le prince Kitashirakawa Yoshihisa (北白)宮能久 親王, 1847-1895), oncle de l'empereur Meiji, dirigea en 1895 la campagne militaire japonaise lors de la conquête de Taïwan et mourut pendant la guerre.

Il fut, dans l'histoire moderne du Japon, le premier membre de la cour à mourir lors d'une expédition militaire. Sous la colonisation japonaise, le prince Yoshihisa fut peu à peu déifié jusqu'à occuper une place prédominante dans le culte shintoïste sur l'île. Dès 1901, le gouverneur Kodama Gentaro fit construire un temple shintoïste sur le site de Yuanshan, à Taipei, afin que le prince Yoshihisa y soit vénéré en tant que principale divinité. Le temple fut le plus important du genre à Taïwan. 


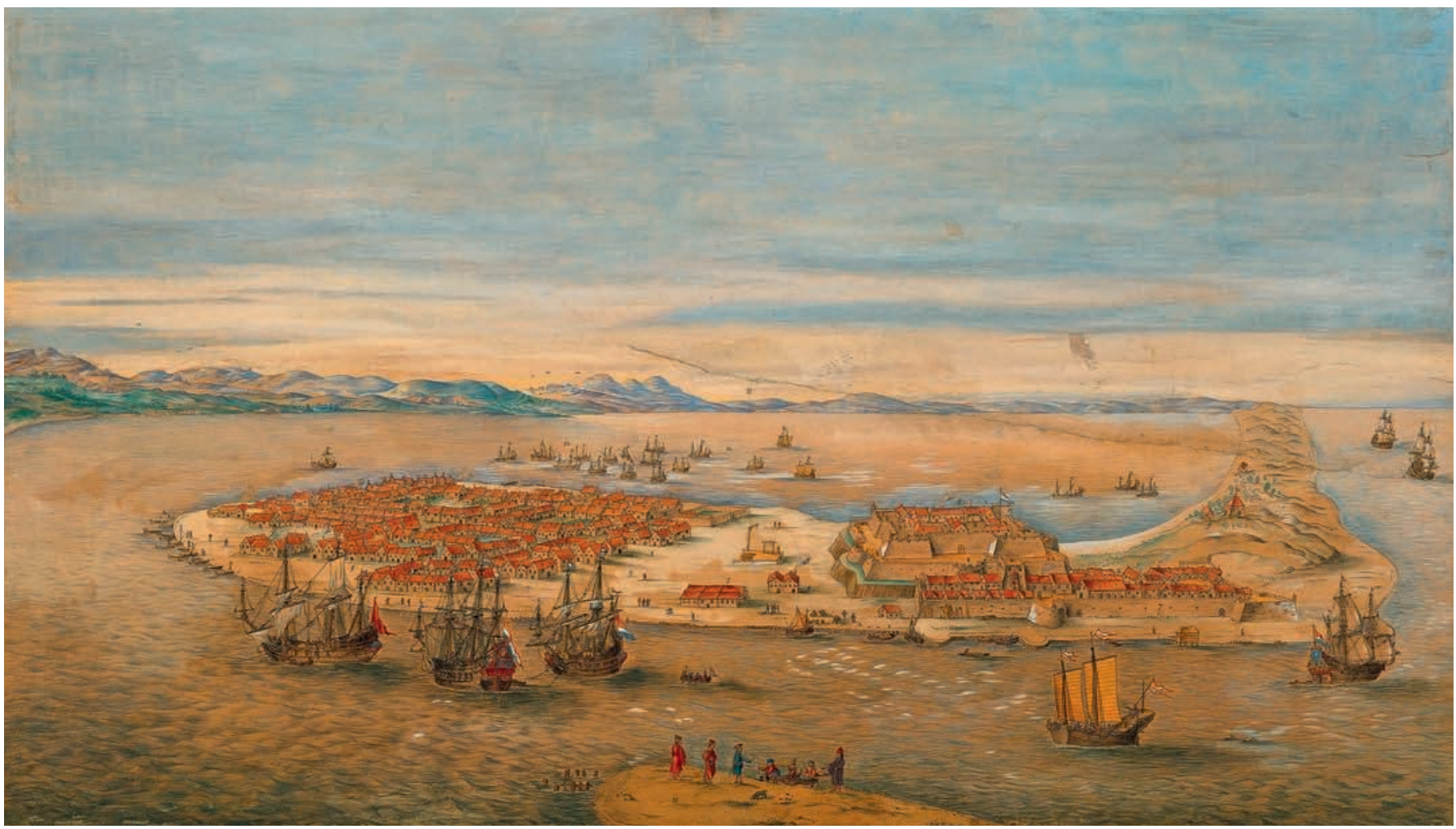

page 64

et ci-dessus

fig. 3

Port de Dayuan (actuel Tainan). D'après une peinture de Johannes Vingboons (1616/1617-1670), commandée vers 1640 par la Compagnie néerlandaise des Indes orientales.

Copie réalisée par

l'Ambassade japonaise à Vienne dans les années 1930, à la demande du musée du Gouverneur

(C) musée national taïwanais. symptomatiquement. La création des petits musées illustre cette situation d'involution du pays, qui se traduisit par une tendance générale à s'emparer de la mémoire communautaire de la république et surtout de l'Empire chinois, astucieusement sacralisé.

L'exaltation d'un nationalisme impérial chinois activement promu sur l'île, qu'il s'agisse de la volonté d'opérer une décolonisation vis-à-vis du Japon ou de la nécessité de s'affirmer face à la prise du pouvoir par le Parti communiste dans l'autre Chine, dont les traditions culturelles se voyaient notoirement menacées de destruction, aboutit à une dynamique bien singulière. L'examen du cas de deux musées locaux de la région de Tainan nous permettra de suivre de plus près, et localement, cette évolution.

Penchons-nous d'abord sur le cas du musée municipal du Patrimoine national de Tainan. Obéissant à l'ordre de commémorer «le tricentenaire de la reconquête de Taïwan par le héros national Cheng Ch'eng-kung ", la municipalité de Tainan entreprit la restauration du temple du vice-roi Yanping ${ }^{5}$ et la construction d'un mémorial dédié à sa gloire. Mais le projet initial de restauration se transforma rapidement en un programme de reconstruction totale: les deux anciens bâtiments, l'un dans le style de l'architecture hoklo (Chine du Sud-Est), l'autre dans celui des temples japonais, furent tout simplement démolis pour laisser place à une construction de style palatial impérial du Nord, achevée en 1964. Le mémorial implanté à proximité fut terminé l'année suivante, et le musée municipal d'Histoire de Tainan fondé par les Japonais dans les années 1930, qui occupait jusqu'alors le pavillon Chikan, y fut transféré. En prévision de ce déménagement, il fut 
d'ailleurs rebaptisé dès 1963 musée municipal du Patrimoine national de Tainan, mettant bien en avant le caractère national et non plus historique de la collection. Ce transfert d'un musée d'histoire locale depuis un lieu de mémoire historique - le pavillon Chikan étant le fort Provintia hollandais du $\mathrm{XVII}{ }^{\mathrm{e}}$ siècle - vers un lieu de culte politico-religieux institué par le pouvoir impérial transforma du même coup un patrimoine culturel civil en un objet de culte au sein d'un panthéon à caractère fortement étatique.

Cette transformation symbolique, probablement imprévue, qui plaçait la culture et l'histoire locales dans un cadre politico-religieux national devint au milieu des années 1970 une démarche adoptée sciemment par le pouvoir, dans l'espoir sans doute de contrer la progression du séparatisme populaire taïwanais. En 1975, le premier musée local créé après la guerre, le musée du Patrimoine historique du district de Tainan, fut installé au palais Ciji - un temple taoïste dédié au culte du Grand Empereur protecteur de la vie, Baosheng Dadi, à Xuejia. Le choix de ce site est loin d'être innocent.

Le palais Ciji se trouvait au centre d'un espace cultuel couvrant un important ensemble de villes et villages de la région ${ }^{6}$. Le 31 du troisième mois de chaque année lunaire, le temple organisait sur le site de Touqianliao, au bord de la mer, lieu présumé du débarquement de l'armée et de la population civile venues avec le général Cheng, un rituel de sacrifice et de vénération à distance à l'adresse du temple Ciji ancestral situé à Baijiao, dans le Fujian, de l'autre côté du détroit de Taïwan. Des études montrent qu'à l'époque coloniale et au début de la dictature nationaliste, il ne restait plus grand chose de ce rituel, né aux environs de 1840. Mais en 1971, prétextant les soixante ans de la fondation de la république de Chine, le palais Ciji de Xuejia organisa une grande fête religieuse avec une procession qui s'étendait sur plus de dix kilomètres, marquée par la participation d'une centaine de chars et de plus de soixante palanquins divins qui firent le tour du site trois jours durant. L'événement attira beaucoup de monde, et le rite du sacrifice à distance, en particulier, impressionna fortement l'équipe chargée des affaires culturelles du comité provincial du Parti nationaliste.

La manière dynamique dont s'articulaient le religieux, le politique et le culturel autour du palais Ciji était en tout point conforme au modèle promulgué à l'époque d'une histoire de Taïwan en lien étroit avec le continent, d'où le choix du lieu, en 1975, pour abriter le nouveau musée. La vivacité du rituel célébrant un lien ainsi sacralisé venait en effet à point nommé pour insuffler une ferveur populaire salutaire dans une représentation étatique bien trop schématique et abstraite. D'ailleurs, le rituel organisé par le palais Ciji, limité au début au seul cercle cultuel autour du temple, s'élargit rapidement dans les années 1970 à tout le territoire du district avant de gagner la province puis la nation elle-même dans les années $1980{ }^{7}$. Cet élargissement constituait une belle illustration de la dimension religieuse des relations entre centre officiel du pouvoir et localités, héritage, là encore, de la période impériale.

\section{Jeux de la démocratisation: le complexe des identités}

Les conflits de plus en plus ouverts entre une identité chinoise et une identité taïwanaise que connut la société dans les années 1980 mirent le nationalisme chinois devant un adversaire autrement plus coriace à l'intérieur
5. Le temple du vice-roi Yanping était un lieu de culte dédié à Cheng Ch'eng-kung. Il fut construit dans les années 1870, après que le gouverneur impérial Shen Baozhen, envoyé sur l'île en 1874, eut fait auprès de la cour l'éloge de ce général, qui avait battu les Hollandais, pour son courage et sa loyauté. Un ordre impérial fut émis, qui autorisa la construction d'un temple et officialisa pour la première fois le statut politique historique de ce personnage. Durant les années japonaises, un temple shintoïste fut également édifié à proximité.
6. Le sinologue Kristofer Schipper, spécialiste du taoïsme, a livré des descriptions des cérémonies cultuelles organisées dans les temples autour de la ville de Tainan, cérémonies auxquelles il avait participé dès les années 1960 et 1970 Deux articles sont repris en français dans son ouvrage La Religion de la Chine.

La tradition vivante (Paris, Fayard, 2008), consacrés précisément au temple Ciji Gong de Xuejia et au culte du Grand Empereur protecteur de la vie.

7. En 1979, le comité d'organisation de la cérémonie s'élargit de la ville de Xuejia à tout le district de Tainan, puis en 1981 ce fut le gouvernement provincial qu prit en charge l'organisation de la cérémonie. En 1989,

celle-ci eut lieu sous la présidence du ministre de l'Intérieur. 
8. Après les premières élections libres du gouverneur de la province (1994) et du président de la République (1996), le recouvrement à $98 \%$ du territoire entre ces deux échelons ainsi que l'inefficacité de la structure administrative à quatre niveaux (république, province, district/municipalité, village/ bourg) provoquèrent de vifs débats dans l'opinion. Face à une situation d'autant plus complexe que l'identité nationale était problématique et les luttes de pouvoir intenses, Lee Teng-hui prit l'initiative d'une réforme administrative appelée "essentialisation de la province»(精省, jingsheng), qui consistait à supprimer à terme cet échelon administratif.

9. Dans le même temps, le musée provincial de Taïwan, l'ancien emblématique musée du Gouverneur, devint le musée national de Taïwan. de l'île, surtout à partir de 1987, quand l'abrogation de la loi martiale mit un terme à un règne autoritaire de près de quarante ans et que Taïwan s'engagea dans un processus de démocratisation. La sphère politique locale vit alors arriver une nouvelle force organisée, opposée au pouvoir central et déterminée à mettre en valeur un imaginaire autre que celui de la grande nation chinoise. En 1989, le tout nouveau Parti démocrate progressiste (PDP), créé illégalement trois ans auparavant, gagna six des vingt et une municipalités lors des élections municipales grâce à sa stratégie dite d'«encerclement du centre par les localités». Dans ce contexte de mutation politique et de mouvements sociaux très actifs, le district d'llan, une des localités les plus militantes, dirigée désormais par le parti d'opposition, se lança dans la création de deux musées locaux: en 1992, le gouvernement du district annonça la constitution du comité préparatoire d'un musée d'histoire locale baptisé musée de Lanyang, puis, en 1993, il ouvrit au public les archives d'llan, premier musée officiel «d'opposition » consacré à l'histoire locale de Taïwan.

En effet, avec le processus de démocratisation, c'était Taïwan dans son ensemble qui se trouvait dans une mouvance générale de redéfinition identitaire. Lee Teng-hui (李登輝, né en 1923), le président successeur de Chiang Ching-kuo (蔣經國, 1910-1988), l'avait bien compris (Lee 1994, 1999). En homme politique avisé, il connaissait l'importance des symboles. Dès 1992, il ordonna la constitution du comité préparatoire du musée provincial d'Histoire de Taïwan. Et à l'issue de sa victoire aux premières élections présidentielles au suffrage universel en 1996, trois autres institutions nationales centrées sur la culture taïwanaise furent mises en chantier: le Centre national des arts traditionnels en 1996, le Centre national de la conservation et de la recherche des patrimoines culturels et le musée national de la Littérature taïwanaise en 1997. Avec la réforme dite d'«essentialisation » de la province ${ }^{8}$ et la disparition de cet échelon administratif sur l'île en 1998, la consécration d'une nouvelle identité taïwanaise, débarrassée de son statut local de «province», était bien désormais de portée nationale.

Cette «délocalisation» de l'identité taïwanaise avait pour objectif évident une «nationalisation" de Taïwan, qui se traduisait, non sans paradoxe, par une relocalisation à travers la communautarisation de la société. Le processus de démocratisation engagé dans les années 1990 était en effet jalonné de mouvements identitaires. La restitution de leur terre et la rectification de son nom, réclamées par les aborigènes stigmatisés jusqu'alors sous l'appellation condescendante de "compatriotes montagnards ", conduisirent à la création du ministère des Affaires aborigènes en 1996. Et un mouvement similaire chez les Hakkas aboutit en 2001 à la création du Comité des affaires hakkas. Une nouvelle structure d'identification communautaire «taïwanaise», multiethnique et multiculturelle, fut ainsi reconnue comme telle au sein même de l'institution politique (Chang 2002).

Dans le domaine de la construction des musées, non seulement le musée provincial d'Histoire de Taïwan, voulu par Lee, fut requalifié musée national ${ }^{\mathbf{9}}$, mais les initiatives locales allant dans le sens de cette reconfiguration furent également avalisées: en 1999, le Yuan exécutif, instance gouvernementale centrale, valida le projet du musée de Lanyang en accep- 

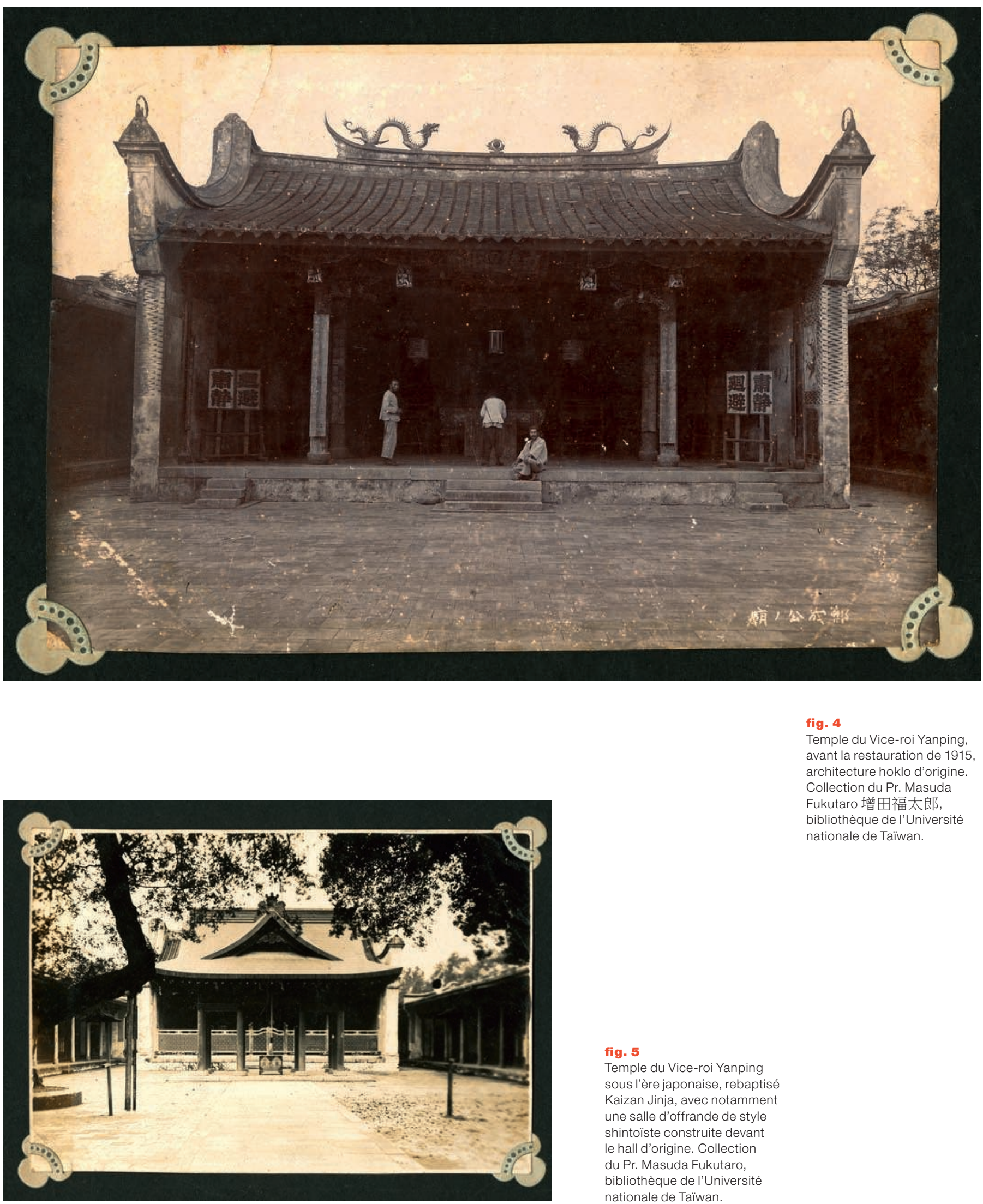

fig. 5

Temple du Vice-roi Yanping sous l'ère japonaise, rebaptise Kaizan Jinja, avec notamment une salle d'offrande de style shintoïste construite devant le hall d'origine. Collection du Pr. Masuda Fukutaro, bibliothèque de l'Université nationale de Taïwan.

fig. 4

Temple du Vice-roi Yanping, avant la restauration de 1915, architecture hoklo d'origine. Collection du Pr. Masuda Fukutaro 增田福太郎, bibliothèque de l'Université nationale de Taïwan. 
10. Musée du Patrimoine hakka de Kaohsiung (1998), musée du Patrimoine hakka de Meinung (2001), musée du Patrimoine hakka de Liudui (2001)

11. Parc culturel hakka dans le district de Taidong (est, comité préparatoire), de Liudui dans le district de Pingdong (sud), de Sanxia dans le district de Taipei (nord), de Dongshi dans le district de Taizhong (centre) et de Tongluo dans le district de Miaoli (ouest) tant de le financer conjointement avec le gouvernement local; d'importantes subventions d'État ont été accordées au musée depuis lors. Et des projets de musées de culture hakka furent conçus sous les auspices du Conseil des affaires culturelles dès $1998{ }^{\mathbf{1 0}}$, avant d'être repris et élargis par le tout nouveau Comité des affaires hakkas dans les années 2000.

La victoire en l'an 2000 du candidat indépendantiste Chen Shui-bian (陳水扁, né en 1950) aux élections présidentielles ne fit que renforcer la tendance. La construction d'une mémoire historique des Taïwanais, ayant comme objectif la consolidation de la nouvelle identité taïwanaise, s'appuya ainsi de plus en plus sur les musées et leur symbolique culturelle. Le musée (désormais) national d'Histoire de Taïwan, par le choix de son site d'implantation et par son style architectural, nous en fournit un exemple des plus éloquents. Construit sur le fameux site du débarquement de l'armée de Cheng Ch'eng-kung dans la ville de Tainan, capitale de l'île depuis l'époque des Hollandais jusqu'à l'arrivée des Japonais, il affiche d'emblée, dans sa conception architecturale, ses caractéristiques taïwanaises. CEuvre de l'architecte Chien Hsueh-yi (簡學義, né en 1954), le corps du bâtiment abandonne le style «impérial »-somptueuses toitures de palais chinois et éléments de décoration classiques - couramment adopté depuis un demisiècle pour les grandes constructions officielles à Taïwan et reprend deux éléments emblématiques des constructions traditionnelles de l'île: les dalles de pierre des maisons aborigènes d'une part et les murs en briques rouges de l'habitat populaire hoklo de l'autre.

Musée né dans une époque démocratique, la nouvelle institution prit bien soin, par le biais d'une muséographie renouvelée, de contourner la délicate question du positionnement politique dans le conflit entre les deux nationalismes en vigueur à Taïwan. Les principaux protagonistes du projet, anthropologues de formation, abandonnèrent la vision traditionnelle d'une histoire dominée par la politique, et optèrent pour un traitement sous l'angle de la quotidienneté populaire et une mise en scène articulée autour de trois grands thèmes: le processus de modernisation de Taïwan, l'évolution des relations intercommunautaires et les échanges de l'île avec le monde extérieur. Toutefois, l'effacement voulu des repères politiques dans le musée ne gomme pas le fait que le musée lui-même est le résultat de l'affirmation de la nouvelle identité taïwanaise, porteuse d'une revendication politique nationale.

La prise en main de la «nationalisation» de Taïwan par le biais de la «relocalisation» dans le domaine de la construction culturelle s'accéléra avec l'arrivée en 2002 à la tête du Yuan exécutif de l'ancien maire du district d'llan, Yu Shyi-kun (游錫等, né en 1948). Plusieurs projets d'envergure furent lancés par le gouvernement pendant les trois années de son mandat. Non seulement le Comité des affaires hakkas annonça le projet de cinq parcs culturels implantés sur tout le territoire 11, mais un programme de maisons de culture locales fut aussi lancé dès 2002, qui envisageait d'étendre la création des musées locaux à l'échelon administratif des districts, avec comme objectif affiché «Un canton, un musée». II préconisait de créer un lieu d'exposition ou de spectacle dans chaque agglomération, offrant aux habitants un accès de proximité à la culture et constituant de nouvelles ressources destinées à dynamiser le tourisme culturel dans les 
régions. En l'espace de quelques années, le nombre de musées consacrés à la culture locale augmenta considérablement, passant d'une centaine à plus de quatre cents. Le nombre de musées communautaires dédiés aux aborigènes atteint par exemple rapidement la cinquantaine (Hu 2007).

\section{Conclusion: absence de la société dans les musées locaux}

Dans l'analyse de la construction des musées à Taïwan, il est convenu de considérer l'année 1987 comme un tournant. Dans la période précédente, que ce soit sous le régime colonial japonais ou sous le régime autoritariste des Chiang, l'entreprise muséale consistait essentiellement à imposer une mémoire politique sans véritable valeur culturelle. Dans la période qui suivit, caractérisée par un processus de transition vers un régime nationaliste démocratique - que ce soit lors de la démocratisation de Lee Teng-hui ou encore dans la pratique du multiculturalisme engagée depuis les années 2000 -, les différents protagonistes de l'entreprise muséale mirent tous en avant la nécessité, pour les musées locaux, de répondre aux besoins sociaux et culturels de l'île et semblèrent s'éloigner d'une pratique étroitement politisée des musées, considérée comme relevant des temps révolus de l'étatisme.

C'est pourtant là une vision erronée de la situation. En effet, de la création initiée en 1978 par Chiang Ching-kuo des centres culturels locaux à travers les villes et les districts au programme «Un canton, un musée » lancé par le Conseil des affaires culturelles en 2002, en passant par la vogue des musées locaux subventionnés par l'État dès la fin des années 1990, nous constatons la persistance de l'intervention politique nationale dans l'espace local. Par cette intervention, l'appareil étatique cherche invariablement à intégrer des imaginaires sociaux hétérogènes dans un plan de gestion global et centralisé.

De prime abord, cette intervention de l'État dans les localités semble avoir assuré aux musées locaux de Taïwan une phase de développement prospère, tant sur le plan pratique qu'en termes de production intellectuelle $\mathbf{1 2}$. Mais à y regarder de plus près, le constat est beaucoup moins enthousiasmant: les initiatives muséales multiculturalistes se sont vite retrouvées dans une impasse, surtout après la rapide retombée des premières émotions liées à la reconnaissance d'identités communautaires jusque-là négligées, voire opprimées. Poussés par des besoins politiques souvent liés aux impératifs électoraux, les projets se multiplièrent de façon incontrôlée, manquant souvent de consistance et de gestion dans la durée. La vague de construction des musées ethniques, en grand nombre et en peu de temps, sous la bannière du multiculturalisme, ne donna que trop de «musées à moustiques» (蚊子館, wenziguan), comme on le regrette souvent à Taïwan. Conscients du problème, des organismes ont pris des initiatives pour y remédier en cherchant à impliquer davantage la population dans les projets. Le ministère des Affaires aborigènes, par exemple, lança en 2007 un programme de vivification des musées aborigènes baptisé “Les grands musées avec les petits》(大館帶小館, daguan dai xiaoguan), en proposant d'utiliser les ressources des collections des grands musées nationaux ${ }^{13}$ pour insuffler une nouvelle dynamique dans la vie des musées et des centres culturels locaux et ethniques ${ }^{\mathbf{1 4}}$. Si certains protagonistes,
12. Avec les programmes nationaux de construction des musées locaux on voit paraître, sur des questions tant techniques que théoriques, quantité de publications dans les médias et le milieu scientifique. La revue scientifique de référence Museology Quarterly par exemple a publié, dès 1993, un numéro spécial consacré au thème "Musée et communautés" (en lien avec le mouvement de la création intégrée de communautés, initié en 1994 par le Conseil des affaires culturelles), et en 2003 un second numéro spécial sur le thème "Musée et interactions communautaires " (en écho à la politique de développement des musées locaux menée en 2002 par le Conseil des affaires culturelles). En 2010 lors de l'inauguration du musée de Lanyang, fut organisé un colloque intitulé Museum Encounter: Experience of Taiwan in Cross-Cultural Perspectives, sur le thème des musées locaux.

13. En collaboration avec le musée national de Taïwan, le musée national des Sciences naturelles, le musée national d'Histoire et le musée nationa de la Culture préhistorique.
14. En 2009, le musée national de Taïwan s'associa à deux musées aborigènes (le musée Kiwit de Hualian et le musée Tayal d'llan) pour une série de manifestations intitulée "Retour à la maison des trésors".

Des représentants des tribus aborigènes furent invités dans les réserves pour y sélectionner un ensemble d'objets que le musée accepta de prêter dans le cadre d'une exposition organisée chez les tribus mêmes. L'événement associait les aborigènes aux prises de décision et des rituels ont été reconstitués pour l'occasion. L'opération s'apparentait à une recomposition de la mémoire collective. Voir Liao 2011. 


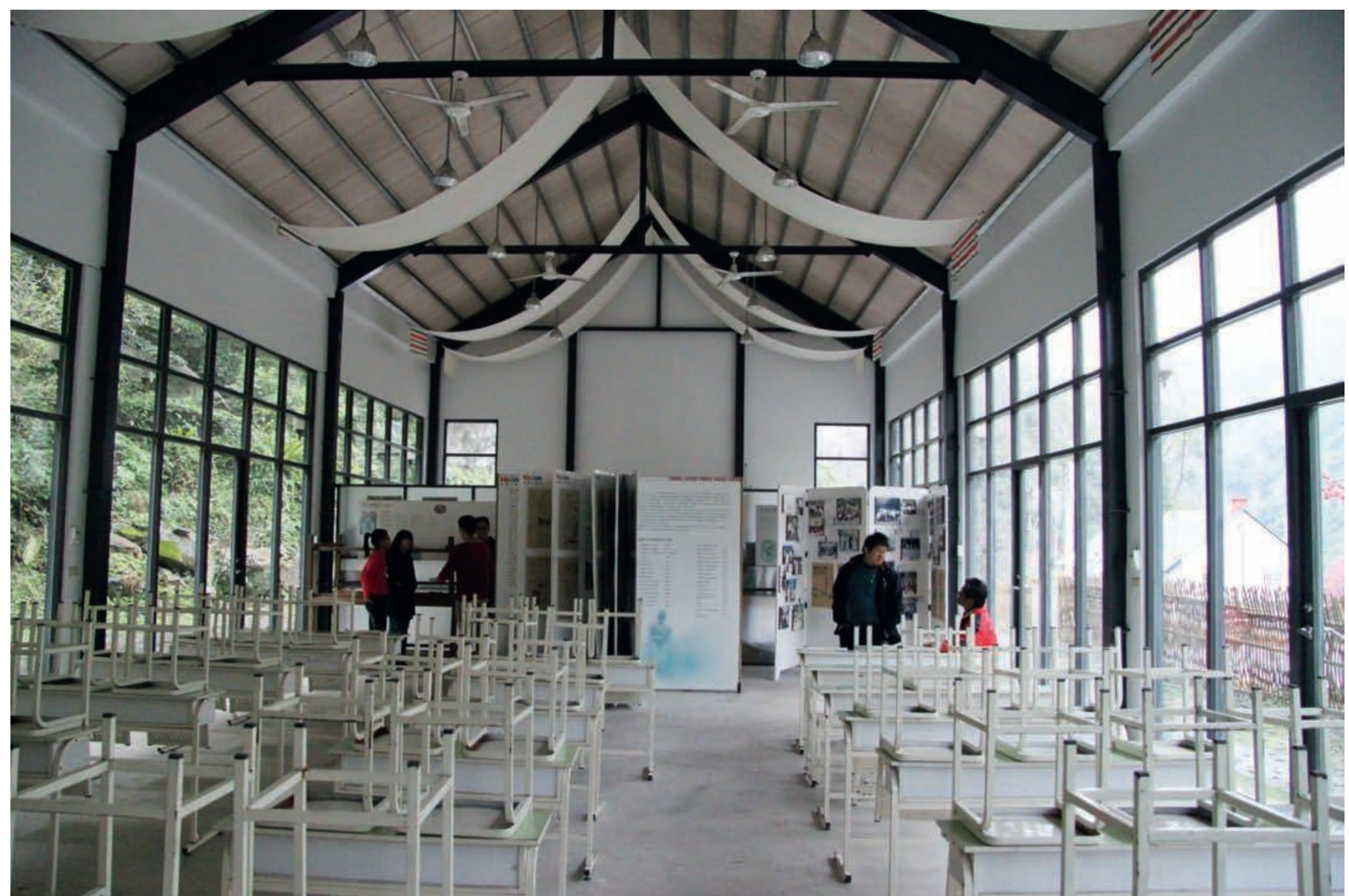

fig. 6

Intérieur d'un «musée à

moustiques » (un musée de

la littérature aborigène dans

une région montagneuse).

Photo Lee Wei-I.

fig. 7

À côté du temple du

Vice-roi Yanping, le Mémorial

de Zheng Chenggong

(Koxinga) ouvert en 2003 ,

se substituant au Musée

municipal du patrimoine

national de Taïnan transféré

dans les locaux en 1965

Photo Lee Wei-I.

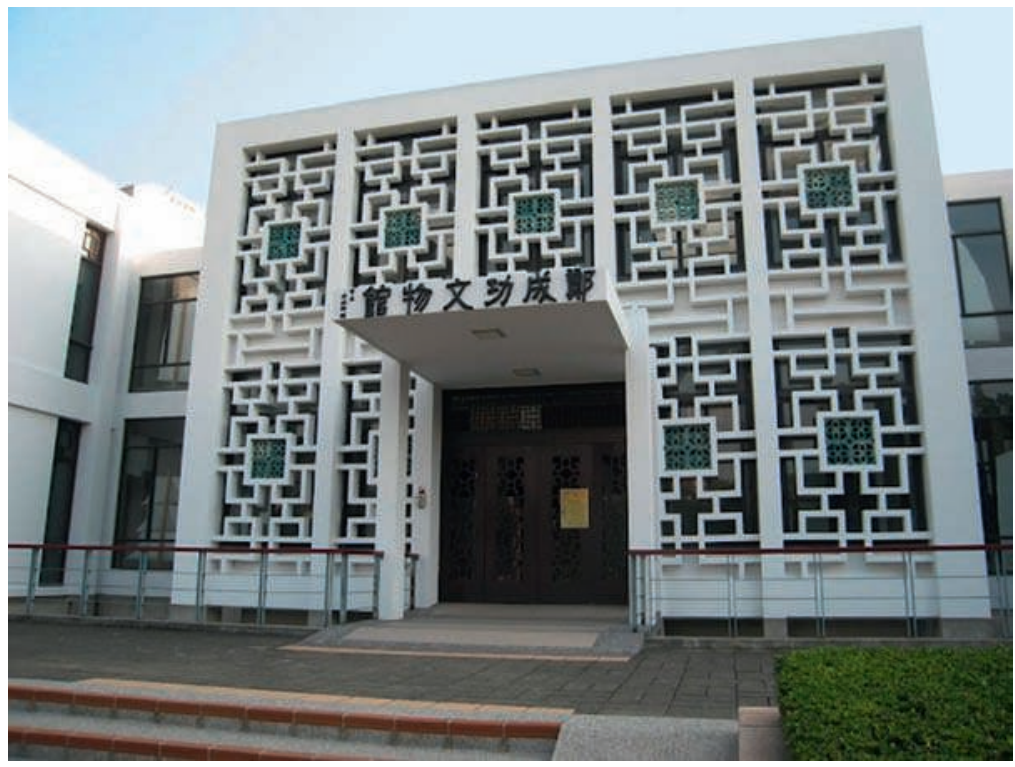


du côté des promoteurs du programme, avaient consciemment endossé la responsabilité de gérants des collections, engagés dans une démarche d'empowerment des communautés sociales, les acteurs locaux, pris dans des problématiques identitaires, ne pouvaient s'empêcher de se mobiliser autour de l'identité essentialiste d'une ethnicité imaginaire, ignorant voire réprimant l'hétérogénéité intrinsèque des communautés concernées.

Nos observations montrent que la troisième vague de création des musées locaux, en pleine démocratisation, relève en réalité toujours d'une politique de mobilisation culturelle étatique, et ne peut être comprise comme le signe d'une autonomisation de la société civile ou d'une décentralisation politique. Les musées restent prisonniers dans leur fonctionnement de la logique identitaire. L'imaginaire collectif à vocation multiculturelle qu'ils étaient censés véhiculer finit toujours par être réintégré dans un ordre unidimensionnel du temps national.

Ce qui nous amène à une dernière remarque sur l'impasse de ce que nous appellerons des «musées communautaires hors société » et des «écomusées sans conscience historique». Pour y voir plus clair, nous pensons qu'il faut revenir à la question de la formation de l'État et de la construction de l'imaginaire culturel. L'absence d'affirmation d'une subjectivité ouverte dans la représentation de la culture locale n'est pas uniquement due à l'héritage malheureux d'un impérialisme chinois, japonais ou occidental, mais bel et bien le signe indicateur de l'inachèvement de la mue sociale de Taïwan en une société démocratique. Nous avons repéré dans la construction des musées locaux de l'époque coloniale japonaise des manœuvres visant à éliminer l'histoire du terroir pour instaurer un sens de la localité sans attache territoriale. Puis nous avons constaté, sous l'ère dictatoriale et autoritariste, le détachement de l'imaginaire populaire local, politisé et "nationalisé» par le parti au pouvoir, de son assise insulaire. Enfin, nous avons cherché à comprendre le jeu politique, dans la mise en représentation de la culture, d'une nationalisation via la relocalisation par communautarisation, toujours en cours dans la société taïwanaise. Ce qui résulte de ces opérations politiques fondamentalement nationalistes, c'est que la "société» n'y est jamais présente, ou plus précisément qu'une société démocratique, au sens moderne d'association des individus libres et égaux intervenant par concertation dans les affaires de la communauté, peine à prendre sa place dans un contexte d'omniprésence de l'État. Qu'il s'agisse des musées communautaires ou des écomusées à la mode, les projets de construction ne s'engagent pas dans la direction d'une démocratie locale et d'une recomposition rationnelle des relations intercitoyennes, mais s'organisent toujours comme des entreprises politiques dont l'initiative reste fermement tenue par le pouvoir.

Ce constat resterait incomplet sans une prise de conscience de la confusion que font différents protagonistes dans leur analyse de la construction des musées locaux à Taïwan entre des concepts tels que le voisinage (鄰里, linli), le quartier (社區, shequ), la communauté (社群, shequ), la localité (地方, difang) ou encore la société (社會, shehui). En effet, depuis 1990, on ne cesse de se référer au concept de musée de voisinage (Chen 2004), inspiré de l'exemple de l'Anacostia Neighborhood Museum fondé 


\section{ci-contre}

fig. 8

Portrait japonisant de Koxinga. Carte postale publiée par le temple dans les années 1920, sceau rouge

"Souvenir de célébration au Kaizan Jinja". Collection du Pr. Masuda Fukutaro, bibliothèque de l'Université nationale de Taïwan. en 1967 aux États-Unis, et à celui de l'écomusée (Chang 2004) emprunté à l'expérience française. Beaucoup insistent sur l'idée que les musées locaux peuvent être une plateforme d'exercice des droits utile à la mise en œuvre d'une justice culturelle au sein d'un régime politique démocratique. Cependant, les lacunes importantes dans la connaissance des conditions sociologiques de ces pratiques muséales et les glissements sémantiques liés à la traduction des concepts font que l'on confond allègrement les concepts de musée local, musée communautaire et musée de voisinage. Les musées locaux à Taïwan, souvent conçus et compris comme des musées communautaires, se présentent pour la plupart d'entre eux comme une sorte de welfare culturel accordé par le pouvoir à la communauté locale ou ethnique. II n'est donc pas étonnant que ces constructions restent tributaires de la politique culturelle étatique, et qu'elles n'aient rien d'un musée de voisinage fondé sur l'idée d'une société civile autonome.

Parmi la centaine de musées communautaires taïwanais, la plupart se définissent en fonction d'un certain traditionalisme ou autour d'une essence identitaire proches de la notion d'indigénisme dont Albert Memmi s'est servi pour décrire la situation des nouveaux États décolonisés (Memmi 1957). Quant au concept de l'écomusée, il devient un cadre conceptuel pour les professionnels intéressés à légitimer l'écologie de loisir et le tourisme culturel, lesquels, sous la pression de l'industrialisation croissante de la culture, se déclinent désormais uniquement sur le mode d'une question économique locale ou nationale. Le retour obsessionnel à la tradition et le recours irréfléchi à l'identité, dépourvus de conscience sociale, amènent à créer des musées «communautaires » teintés d'un sectarisme fermé, incapables de se constituer en véritable espace social ouvert aux interactions citoyennes. L'attention focalisée sur l'enjeu économique de la construction des écomusées ne fait de ces derniers qu'un slogan publicitaire de plus, en contradiction flagrante avec l'idée même qu'ils sont censés incarner. Tout ceci nous semble bien témoigner d'une terre encore hantée par le colonialisme et l'autoritarisme, où les problèmes rencontrés par ces musées locaux ne sont que le reflet d'une démocratie inachevée.

Institut d'anthropologie Université Nationale de Tsing Hua, Hsinchu, Taïwan. musee.tw@gmail.com 


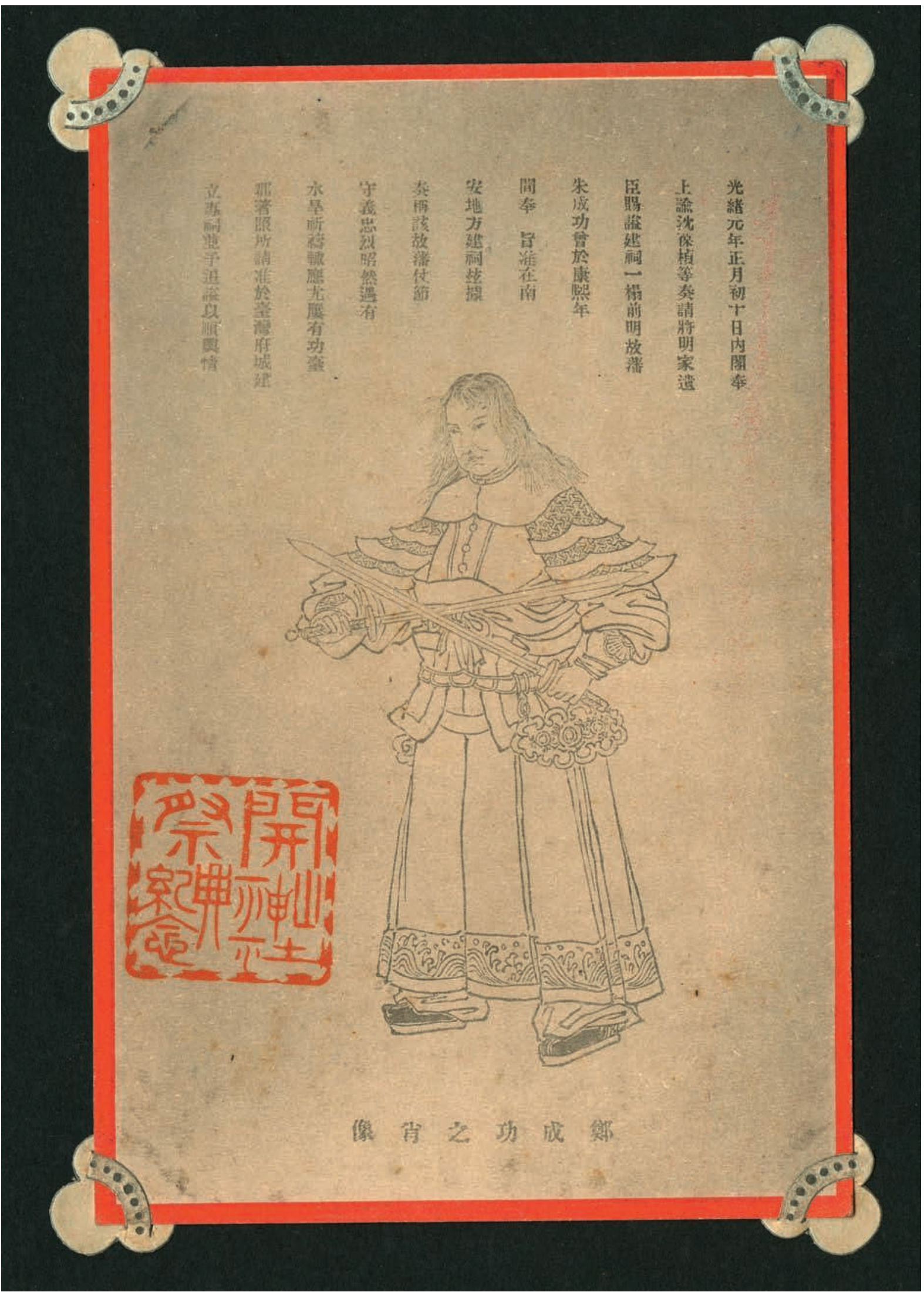




\section{Anderson, Benedict}

1991 Imagined Communities. Reflections on the Origin and Spread of Nationalism. Londres, Verso.

\section{Chang, Mau-Kuei (張茂桂)}

2002 «Taïwan est-il un pays multiculturel?»(台灣是

多元文化國家), Cultural Studies Monthly 13: 1; www.ncu.edu. tw/ eng/csa/journal/journal_ park86.htm.

\section{Chang, Yui-Tan (張譽騰)}

2004 Écomusée: émergence d'un mouvement culturel (生態博 物館：一個文化運動的興起). Taipei, Wouguan Yishu.

\section{Chen, Jia-Li (陳佳利)}

2004 « Mouvement des musées de voisinage: perspective globale» (社 區博物館運動：全球化的觀點), Museology Quartely (博物館學季刊) 18(4) : 43-57.

\section{Chou, Wan-Yao (周婉穷)}

1997 «L'enseignement du savoir technique, l'amour de la localité et l'identité nationale. Une analyse du manuel de la langue nationale dans l'école publique à Taïwan pendant la période de la gestion japonaise" (實學教育、鄉土愛與 國家認同一日治時期臺灣公學 校第三期「國語」教科書的分析), Recherches sur l'histoire taïwanaise (臺灣史研究) 4(2) : 7-55.

\section{Collomb, Gérard}

1999 «Ethnicité, nation, musée, en situation postcoloniale ", Ethnologie française, vol. xxIx, $n^{\circ} 3$ : 333-336 ( $n^{\circ}$ spécial Musée, nation: après les colonies).

\section{Hu, Chia-Yu (胡家瑜)}

2007 «Musée, anthropologie et expositions aborigènes: transformation du champ de représentation culturelle dans le processus historique” (博物館、 人類學與原住民展示一歷史過程 中文化再現場域的轉形變化) The Bulletin of the Department of Archaeology and Anthropology (考古人類學刊) [université de Taïwan] 66: 94-124.

\section{Kaplan, Flora Edouwaye S.}

1994 Museums and the Making of "Ourselves": The Role of Objects in National Identity. Londres et New York,

Leicester University Press.

\section{Lee, Kuo-Hsuan (李國玄)}

2006 Étude sur le développement de la muséologie moderne et le mouvement de protection des patrimoines culturels à Taïwan dans la période japonaise (日治時期臺灣近代博物館學發 展與文化資產保存運動之研究). Zongli, département de l'Architecture de l'université de Chungyuan, mémoire de master.

\section{Lee, Teng-hui (李登輝)}

1994 Gouverner le Grand Taïwan (經營大臺灣). Taipei, Yuanliu.

1999 Revendications de Taïwan (臺灣的主張). Taipei, Yuanliu.

\section{Lee, Wei-I (李威宜)}

2007a "Cent ans de musées à Taïwan. Du colonialisme au nationalisme, vers un imaginaire au-delà de l'identité ? ",

Transcontinentales 4: 145-167.

2007b «The construction of community imaginaries in Taïwan's Museums and Archives Committees (1945-1978) ", in Carsten Storm et Mark Harrison (éd.), The Margins of Becoming Identity and Culture in Taïwan. Wiesbaden, Harrassowitz: 173-184.

2008 Institution des imaginaires collectifs et institutions de mémoire à Taïwan. Aux origines d'une composition identitaire éclatée (1945-1978). Paris, École des hautes études en sciences sociales, thèse de doctorat.

\section{Li, Tzu-Ning (李子寧)}

1997 «Colonialisme et musée. L'exemple du musée du Gouverneur à l'époque de l'occupation japonaise»(殖民主義與博物館一以日 據時期臺灣總督府博物館為例), Les Annales du musée provincial de Taïwan (臺灣省立博物館年刊) 40: 241-273.

\section{Liao, Pei-Shan (廖珮珊)}

2011 Personnes et objets en mouvement: rapport de pouvoir dans les réseaux. Expositions Retour à la maison des trésors du programme "Les grands avec les petits》(移動的人與物：大館 帶小館「文物回娘家」特展 網 絡的權力關係). Hsinchu, Institut d'anthropologie de l'université de Tsing Hua, mémoire de master.

\section{Lu, Shao-Li (呂紹理)}

2005 Montrer Taïwan: représentation figurative du pouvoir, de l'espace et de la domination coloniale (展示臺灣：權力、空 間與殖民統治的形象表述). Taipei, Maitian.

\section{Macdonald, Sharon}

2003 «Museums, National Postnational and Transcultural Identities ", Museum and Society 1(1): 1-16.

\section{Memmi, Albert}

1957 The Colonizer and the Colonized. Boston, Beacon.

\section{Poulot, Dominique}

1997 Musée, nation patrimoine 1789-1815.

Paris, Gallimard.

\section{Schipper, Kristofer}

2008 La Religion de la Chine. La tradition vivante. Paris, Fayard.

\section{Thiesse, Anne-Marie}

1999 La Création des identités nationales: Europe $x v 1 I^{e}-x x^{e}$ siècle. Paris, Seuil.

Ts'ai, Hsiang-Hui (蔡相煇)

1984 " Le temple de deux rois et les tombes du père et du fils Zheng " (二王廟與鄭成功父子陵寢), Revue de la renaissance culturelle et artistique (文藝復興月刊) 158: $42-49$

ci-contre

Port de Dayuan (actuel Tainan). D'après une peinture de Johannes Vingboons (1616/1617-1670), commandée vers 1640 par la Compagnie néerlandaise des Indes orientales. Copie réalisée par l'Ambassade japonaise à Vienne dans les années 1930, à la demande du musée du Gouverneur (c) musée national taïwanais. 

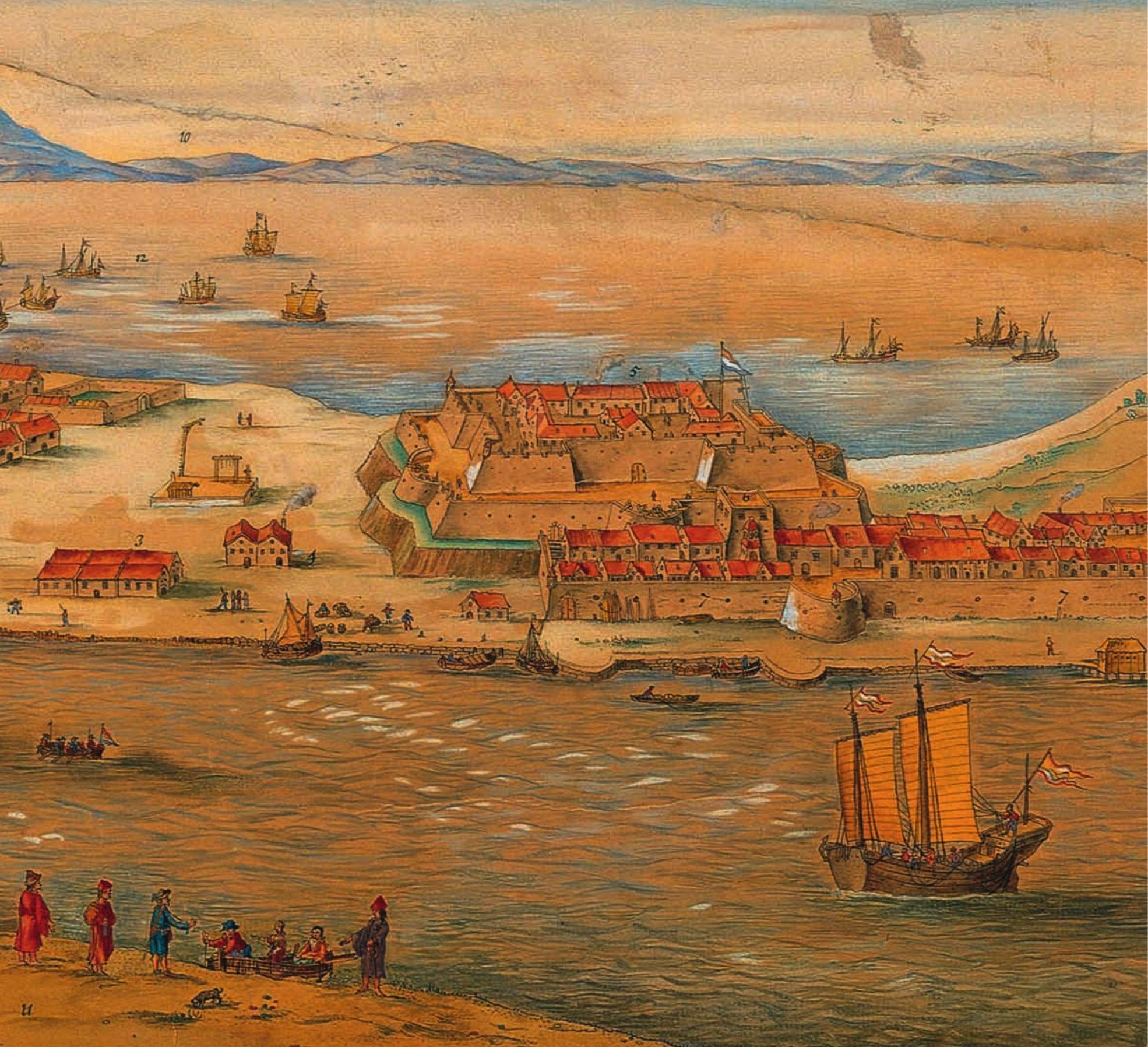